\title{
PARVIMONAS MICRA AND FUSOBACTERIUM NUCLEATUM SEPTIC ARTHRITIS: A RARE ANAEROBIC DOUBLE TROUBLE
}

\author{
PADMAJA ANANTH SHENOY ${ }^{1 *}$, ROHIT GUPTA ${ }^{1}$, SHASHIDHAR VISHWANATH ${ }^{1}$, MONAPPA A NAIK ${ }^{2}$ \\ SEEMA SHETTY ${ }^{1}$, KIRAN CHAWLA ${ }^{1}$
}

${ }^{1}$ Department of Microbiology, Kasturba Medical College, Manipal Academy of Higher Education, Manipal, Karnataka, India. ${ }^{2}$ Department of Orthopaedics, Kasturba Medical College, Manipal Academy of Higher Education, Manipal, Karnataka, India. Email: padmaja.shenoy@manipal.edu

Received: 14 July 2018, Revised and Accepted: 22 August 2018

\begin{abstract}
Septic arthritis is a condition initiated by pathogenic inoculation of joints either by direct or hematogenous route, necessitating immediate medical attention. Among aerobic bacteria, Staphylococcus aureus and Streptococcus spp. are commonly found in association with septic joints. Anaerobes are very rarely involved in the causation of septic arthritis with an estimated rate of $<1 \%$. We are presenting a case of septic arthritis of knee joint by Parvimonas micra and Fusobacterium nucleatum, both being constituents of microbial flora in the oral cavity and gastrointestinal tract. Gram stain and anaerobic culture incorporated along with the aerobic culture of synovial fluid have played an important role in the preliminary diagnosis of anaerobic septic arthritis in this case.
\end{abstract}

Keywords: Septic arthritis, Anaerobic bacteria, Gram stain.

(c) 2019 The Authors. Published by Innovare Academic Sciences Pvt Ltd. This is an open access article under the CC BY license (http://creativecommons. org/licenses/by/4. 0/) DOI: http://dx.doi.org/10.22159/ajpcr.2019.v12i1.28499

\section{INTRODUCTION}

Septic arthritis or infective arthritis is a condition initiated by pathogenic inoculation of joints either by direct or hematogenous route accounting to significant morbidity and mortality $[1,2]$. Bacterial pathogens are commonly associated with invasion of joints; however, viral, fungal, and mycobacterial agents are also linked with septic arthritis [3]. Among the bacterial agents, aerobes are considered to be frequent isolates because of their rapid destructive nature while, the frequency of anaerobic bacteria causing septic arthritis is very low with an estimated rate of $<1 \%[1,4]$. Among the aerobic bacteria, Staphylococcus aureus and Streptococcus spp. account for more than $90 \%$ of the cases [5] and Gram-negative bacteria are more common in elderly patients and immunocompromised individuals [1,3]. We present here a case of septic arthritis of knee joint by Parvimonas micra and Fusobacterium nucleatum which are constituents of normal microbial flora in the oral cavity and gastrointestinal tract of human beings.

\section{CASE REPORT}

A 48-year-old man presented to the orthopedics specialty with complaints of pain and swelling of the right knee joint for 20 days duration. Pain in the joint was insidious in onset and gradually progressive in nature and was associated with difficulty in walking. The pain worsened over the next 2 weeks with difficulty in extending the knee joint. He complained of high-grade fever and chills which relieved on taking medications. He also complained of pain in the right calf muscle for 5 days before his presentation to the clinic. $\mathrm{He}$ was not a known diabetic. There was no history of trauma, previous inflammatory joint disease, ear infection/respiratory tract infection, or any prior dental procedures. On local examination, right knee joint was warm with the presence of an effusion. Tenderness was elicited with a fixed flexion deformity of $40^{\circ}$. The range of motion was restricted to $40-70^{\circ}$ with pain throughout.

At admission, blood picture showed hemoglobin of $10 \mathrm{~g} / \mathrm{dl}$, total leukocyte count of $10 \times 10^{3} / \mu$ l (neutrophils, $82.6 \%$ ), and erythrocyte sedimentation rate (ESR) was $120 \mathrm{~mm} / \mathrm{h}$. Absolute neutrophil count was
$11.2 \times 10^{3} / \mu \mathrm{l}$. Serum C-reactive protein (CRP) was raised $(154.3 \mathrm{mg} / \mathrm{l})$, and plasma lactate levels were in normal limits $(7.5 \mathrm{mg} / \mathrm{dl})$. X-ray of the right knee joint showed early arthritis changes with fixed flexion deformity along with increased soft tissue shadow in the right knee joint. MRI of the right knee joint was suggestive of septic arthritis with effusion in the knee joint and multiple air foci in the suprapatellar bursa. The right knee joint was aspirated and specimens were sent for pathological and microbiological workup. The patient was empirically started on intravenous amoxicillin-clavulanic acid $(1.2 \mathrm{~g} / 12 \mathrm{~h})$ and clindamycin $(600 \mathrm{mg} / 8 \mathrm{~h})$. The analysis of cell counts of aspirated fluid could not be performed due to the presence of degenerated cells in the necrotic background.

The Gram stain of the aspirate fluid showed numerous pus cells with moderate fusiform Gram-negative bacilli and few Gram-positive cocci in pairs. The aerobic cultures were sterile after $72 \mathrm{~h}$ of incubation, while anaerobic cultures yielded the growth of $F$. nucleatum and P. micra. The bacterial identification was confirmed by matrix-assisted laser desorption ionization-time-of-flight mass spectrometry (Vitek MS, bioMérieux Inc.). Both $F$. nucleatum (metronidazole minimum inhibitory concentration $[\mathrm{MIC}] \quad 0.032 \mu \mathrm{g} / \mathrm{ml}$; clindamycin $\mathrm{MIC}$ $0.25 \mu \mathrm{g} / \mathrm{ml}$ ) and P. micra (metronidazole MIC $0.047 \mu \mathrm{g} / \mathrm{ml}$; clindamycin MIC $0.016 \mu \mathrm{g} / \mathrm{ml}$ ) were susceptible to metronidazole and clindamycin by antimicrobial gradient diffusion method (E test, bioMérieux Inc.). Aerobic blood cultures were sterile after 5 days of incubation. Following the microbiological culture reports, intravenous antibiotics were changed to ceftriaxone $(2 \mathrm{~g} / \mathrm{d})$ and (clindamycin $600 \mathrm{mg} / 8 \mathrm{~h})$.

In view of his right calf muscle tenderness, Doppler ultrasound of right leg was performed which revealed the presence of echogenic thrombus in the femoral vein and popliteal vein. Cardiology consultation was sought and was advised for prophylactic inferior vena cava (IVC) filter insertion to prevent the dislodgement of the thrombus. After 3 days of IVC filter insertion procedure, the patient underwent right knee arthroscopic debridement, and intraoperative soft tissue and synovial fluid specimens were sent for bacteriological analysis. No significant growth was observed in aerobic and anaerobic cultures. 
The post-operative period was uneventful, and he was mobilized with partial weight bearing. The patient was discharged with oral cefuroxime for 6 weeks. He was symptomatically better in his review visits, and results of the absolute neutrophil count, CRP, and ESR were within normal limits 6 weeks after his discharge.

\section{DISCUSSION}

Septic arthritis constitutes a true rheumatologic emergency requiring immediate medical attention and any delay in treatment may result in irreversible joint damage [6]. Hence, timely implementation of targeted antimicrobial therapy is the need of the hour to knock down the mutilation of joints $[7,8]$. Septic arthritis frequently involves larger joints, more than $60 \%$ of the cases being associated with knee joints. Involvement of smaller joints is common in patients with pre-existing inflammatory joint diseases [1]

The diagnosis of bacterial arthritis can be made in patients with mono or oligoarticular arthritis fulfilling any one of the four criteria of widely recognized case definition proposed by Newman: (1) Isolation of an etiological agent from an affected joint, (2) isolation of an etiological agent from another source in context of swollen, hot joint, (3) clinical features and turbid joint fluid in the presence of previous antibiotic therapy, and (4) histologic or radiologic evidence consistent with septic arthritis [6-8].

Among the diverse pathogens associated, aerobic bacteria are the major culprits in cases of septic arthritis, while, anaerobes are reported in very rare instances [1]. P. micra a fastidious anaerobic Gram-positive cocci, previously known as Peptostreptococcus micros, has recently been reclassified within the Parvimonas genus [9]. P. micra is predominantly isolated from dental, intra-abdominal infections and skin and soft tissue abscesses, particularly in intravenous drug users [10]. F. nucleatum is a fusiform, non-sporing, anaerobic, Gram-negative bacilli, commonly isolated from periodontal diseases, mastoiditis, peritonsillar, and retropharyngeal infections [11].

Diverse risk factors, namely trauma, diabetes mellitus, prosthetic joints, gout, intravenous drug usage, previous surgeries, and history of corticosteroid injections play key roles in the causation of joint sepsis [1,7]. P. micra being a oral commensal has been reported in bone and joint infections following dental procedures or periodontal infections [12]. Interestingly, no such risk factors were observed in the present case, and blood cultures also remained sterile.

Positivity rate of Gram stain from synovial fluid accounts for about $50 \%$; however, the absence of bacteria in microscopy does not rule out septic arthritis considering the prior intake of antibiotics [1,3]. Gram stain from synovial fluid has played a significant role in the preliminary diagnosis of anaerobic septic arthritis in the present case. Aerobic culture remained negative while anaerobic culture yielded $P$. micra and $F$. nucleatum. Polymicrobial anaerobic infection with $P$. micra and $F$. nucleatum is a rare finding in bone and joint infections, except for a case of spondylodiscitis reported by Cleaver et al. [13]. Elevated counts of white blood cells in synovial fluid have little significance in the diagnosis of septic arthritis; however, in the present case, the procedure could not be performed in the presence of necrotic cells. Increased absolute neutrophil count in blood was noted

Wide usage of metronidazole as the single antimicrobial has been stated in previous literature [14] and it remains an empirical drug of choice for most of the anaerobic infections with minimal resistance rates whereas, resistance to clindamycin is emerging globally [15]. Both the isolates, in this case, were susceptible to clindamycin; thus, the patient showed good clinical response.

\section{CONCLUSION}

The timely initiation of appropriate antimicrobial therapy combined with a surgical clearance of purulent material from the infected site aided in the gradual improvement of joint sepsis in this case. Although isolation of anaerobes from septic joint fluids occurs in very rare instances, their importance should never be underestimated, as delayed or inadequate treatment can result in irreversible joint destruction eventually leading to disability. This case report brings in a need to look at septic arthritis investigations in a new light by both clinicians and microbiologists alike.

\section{AUTHOR'S CONTRIBUTION}

Dr. Padmaja Ananth Shenoy reporting microbiologist, preparation of manuscript, Dr. Rohit Gupta assisted in providing the clinical details of the case, Dr. Shashidhar Vishwanath supervised the manuscript preparation and reviewed the manuscript, Dr. Monappa Naik treating doctor, reviewed the manuscript, Mrs. Seema Shetty organizing the manuscript, and Dr. Kiran Chawla reviewed the manuscript.

\section{CONFLICTS OF INTEREST}

The authors declare no conflicts of interest.

\section{REFERENCES}

1. Mathews CJ, Kingsley G, Field M, Jones A, Weston VC, Phillips M, et al. Management of septic arthritis: A systematic review. Postgrad Med J 2008;84:265-70.

2. Hassan AS, Rao A, Manadan AM, Block JA. Peripheral bacterial septic arthritis: Review of diagnosis and management. J Clin Rheumatol 2017;23:435-42.

3. Hindle P, Davidson E, Biant LC. Septic arthritis of the knee: The use and effect of antibiotics prior to diagnostic aspiration. Ann R Coll Surg Engl 2012;94:351-5.

4. Carrasco Cubero C, Zamora Red P, Maestrojuan JJ, Prieto MD. Septic arthritis due to Fusobacterium nucleatum in an immunocompetent patient. Reumatol Clin 2012;8:98-9.

5. Dutta S, Bishayi B. Effects of ciprofloxacin in combination with either aminoguanidine or meclofenamic acid in modulating S. aureus induced septic arthritis in mice. Int J pharm Pharm Sci 2015;7:355-61.

6. Sharff KA, Richards EP, Townes JM. Clinical management of septic arthritis. Curr Rheumatol Rep 2013;15:332.

7. Gupta MN, Sturrock RD, Field M. Prospective comparative study of patients with culture proven and high suspicion of adult onset septic arthritis. Ann Rheum Dis 2003;62:327-31.

8. Nel JM, Visser A, Visser HF, Goller K, Goller R. Adult septic arthritis in a tertiary setting: A retrospective analysis. SA Orthop J 2009;8:53-8.

9. Tindall BJ, Euzéby JP. Proposal of parvimonas gen. Nov. And quatrionicoccus gen. Nov. As replacements for the illegitimate, prokaryotic, generic names Micromonas murdoch and shah 2000 and Quadricoccus maszenan et al 2002, respectively. Int J Syst Evol Microbiol 2006;56:2711-3.

10. Murdoch DA. Gram-positive anaerobic cocci. Clin Microbiol Rev 1998;11:81-120.

11. Citron DM. Update on the taxonomy and clinical aspects of the genus Fusobacterium. Clin Infect Dis 2002;35:S22-7.

12. Roy M, Roy AK, Ahmad S. Septic arthritis of knee joint due to Parvimonas micra. BMJ Case Rep 2017;2017: pii: bcr-2017-221926.

13. Cleaver LM, Palanivel S, Mack D, Warren S. A case of polymicrobial anaerobic spondylodiscitis due to Parvimonas micra and Fusobacterium nucleatum. JMM Case Rep 2017;26:4

14. Gor AP, Ajbani A, Dalal K. Use of fixed dose combinations of antibiotics in a surgical department of a tertiary care teaching hospital. Int J Pharm Pharm Sci 2015;7:259-62.

15. Loyola-Rodriguez JP, Garcia-Cortes JO, Martinez-Martinez RE, Patiño-Marin N, Martinez-Castañon GA, Zavala-Alonso NV, et al. Molecular identification and antibiotic resistant bacteria isolated from primary dentition infections. Aust Dent J 2014;59:497-503. 